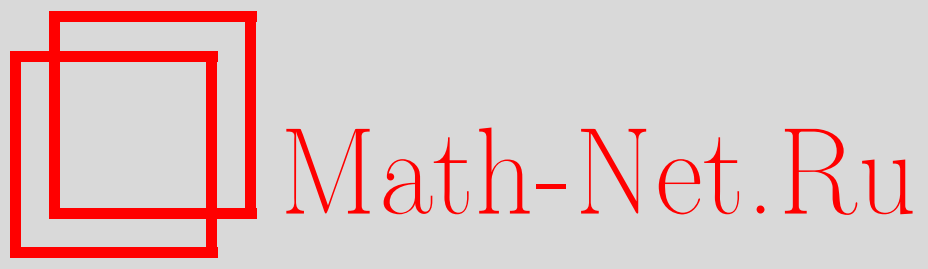

Ю. С. Ильяшенко, Д. А. Рыжов, Д. А. Филимонов, Захват фазы для уравнений, описывающих резистивную модель джозефсоновского перехода, и их возмущений, Функи. анализ и его прил., 2011, том 45, выпуск 3, 41-54

DOI: https://doi.org/10.4213/faa3045

Использование Общероссийского математического портала MathNet.Ru подразумевает, что вы прочитали и согласны с пользовательским соглашением http://www . mathnet.ru/rus/agreement

Параметры загрузки:

IP : 54.210 .77 .194

26 апреля 2023 г., 17:06:46

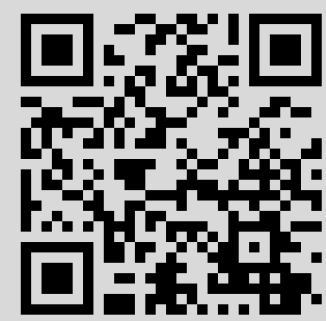




\title{
Захват фазы для уравнений, описывающих резистивную модель джозефсоновского перехода, и их возмущений*
}

\author{
(c) 2011. Ю. С. Ильяшенко, Д. А. Рыжов, Д. А. Филимонов
}

Памяти В. И. Арнольда, великого ученого и учителя

\begin{abstract}
В работе исследуются динамические системы на торе, моделирующие явление Джозефсона в физике сверхпроводников, а также возмущения этих систем. Показано, что в семействе уравнений, описывающих резистивную модель джозефсоновского перехода, захват фазы происходит только при целых числах вращения, и предложен простой способ вычисления границ соответствующих языков Арнольда. Эта часть представляет собой упрощение уже известных результатов о так называемом квантовании числа вращения [4]. Кроме того, мы показываем, что квантование числа вращения только в целых точках представляет собой явление коразмерности бесконечность. А именно, бесконечное множество независимых возмущений порождает счетное число зон захвата фазы, расположенных недискретно.
\end{abstract}

\section{$\S 1$. Введение}

1.1. Формулировка результатов. В работе исследуется семейство дифференциальных уравнений на торе $\mathbb{T}^{2}=\mathbb{R}^{2} / 2 \pi \mathbb{Z}^{2}$

$$
\dot{x}=\sin x+a+\varepsilon f(t)+\delta g(x),
$$

где функции $f$ и $g 2 \pi$-периодические, причем функция $g(x)$ нечетна.

При $\delta=0$ семейство (1) превращается в уравнение

$$
\dot{x}=\sin x+a+\varepsilon f(t),
$$

которое мы будем рассматривать не только при малом, но и при любом $\varepsilon$. Это уравнение используется для моделирования динамики перехода Джозефсона ([7], [9], [11]), и его свойства изучаются как в этом контексте ([2]-[4], [8], [13]), так и при решении других задач ([10], [12]). Мы будем называть его уравнением класса Д. Физическая интерпретация этого уравнения обсуждается ниже.

Отображение Пуанкаре для уравнения (2) обозначим через $P$. Пусть $\lambda=$ $\lambda(a, \varepsilon, \delta):=\lim _{n \rightarrow \infty} \frac{\widetilde{P}^{n}(x)-x}{2 \pi n}-$ его число вращения. Здесь $\widetilde{P}-$ поднятие отображения Пуанкаре на универсальную накрывающую. Соответственно всюду далее число вращения понимается не как элемент из $S^{1}$, а как элемент из $\mathbb{R}$, и на окружности длины $2 \pi$ уравнение $\dot{\varphi}=\lambda$ имеет число вращения $\lambda$. В типичных конечнопараметрических семействах диффеоморфизмов окружности число вращения принимает каждое рациональное значение в целой области пространства параметров. Такие области называются языками Арнольда или зона-

*Работа поддержана грантами NSF 0700973, РФФИ 10-01-00739-а и РФФИ-CNRS 1001-93115-НЦНИЛ_а. Исследования второго автора были выполнены при поддержке Лаборатории им. П. Л. Чебышева Санкт-Петербургского государственного университета, грант Правительства РФ №11.G34.31.0026. 
ми захвата фазы. Однако для уравнений класса Д структура языков Арнольда резко отличается от типичной.

Теорема 1. Захват фазы в семействе уравнений (2) происходит только при целых значениях числа вращения.

Этот результат получен в [4] и в неопубликованных лекциях [6]. Близкие результаты содержатся в работе [13]. В нашем изложении мы следуем лекциям [6].

Дискретность зон захвата фазы в семействе (2) связана с тем, что отображение Пуанкаре уравнения класса Д аналитически эквивалентно дробнолинейному. Когда это отображение является гиперболическим (имеет две неподвижные точки на окружности), число вращения целое, а параметры принадлежат зоне захвата фазы. Когда это отображение параболическое (неподвижные точки сливаются), параметры выходят на границу зоны захвата. Когда отображение Пуанкаре эллиптическое (неподвижные точки уходят в комплексную область), оно приводится к повороту дробно-линейным преобразованием независимо от того, рационально число вращения или нет.

Захват фазы в семействе (1) происходит совсем иначе и во многом напоминает захват в типичных конечнопараметрических семействах. Как показано ниже, в разд. 5.3, для исследования семейства (1) достаточно изучить семейства

$$
\dot{x}=\sin x+a+\varepsilon \sin l t+\delta \sin k x
$$

для различных целых $l$ и $k$. Наша основная цель - описание зон захвата фазы в семействе (3): теоремы 2 и 3 и гипотеза 1 ниже. Чтобы их сформулировать, рассмотрим семейство (3) как малое возмущение интегрируемого семейства

$$
\dot{x}=\sin x+a, \quad x \in S^{1} .
$$

Напомним, что $\lambda=\lambda(a, \varepsilon, \delta)$ - это число вращения отображения Пуанкаре системы (3). При $|a| \leqslant 1$ уравнение (4) имеет особые точки и $\lambda(a, 0,0)=0$. Поэтому всюду ниже будем считать, что $|a|>1$. В этом случае, как показано в $\S 3, \lambda(a, 0,0)=\sqrt{a^{2}-1}$. Область захвата фазы (язык Арнольда) в системе $(3)$, соответствующая числу вращения $\lambda=p / q$, - это множество $T_{p / q}=$ $\{(a, \varepsilon, \delta) \mid \lambda(a, \varepsilon, \delta)=p / q\}$ при условии, что в любой окрестности точки $(a=$ $\left.\sqrt{p^{2} / q^{2}+1}, 0,0\right)$ внутренность этого множества непуста.

Теорема 2. B семействе (3) при любом $l$ u $|k|=2$ происходит захват фазы для любого рационального числа вращения $p / q$ nри $p= \pm l$ u $|q|>1$. Соответствующие языки Арнольда имеют ширину порядка $\varepsilon \delta$.

Последняя фраза означает, что при достаточно малых $\varepsilon, \delta$ сечение языка Арнольда $T_{\lambda}$ прямой $\{\varepsilon, \delta=$ const $\}$ имеет длину, заключенную между $C_{1} \varepsilon \delta$ и $C_{2} \varepsilon \delta$, где $C_{i}=C_{i}(\lambda)$ - положительные константы.

При $|k|>2$ мы умеем доказывать несколько более слабый результат.

Теорема 3. B семействе (3) при любом $l$ u $|k|>2$ происходит захват фазы для любого рационального числа вращения $p / q$ при $p= \pm l$ и всех $q$, кроме, бъть может, $14 k+20$ значений. Соответствующие языки Арнольда имеют ширину порядка єб.

Гипотеза 1. В семействе (3) при любом $l$ u $|k|>1$ nроисходит захват базы для любого рационального значения числа вращения. 
Теоремы 2 и 3 доказаны методами теории возмущений. Доказательства заканчиваются, когда удается показать, что в соответствующих рядах появляется резонансный моном с ненулевым коэффициентом. Этот коэффициент в условиях теоремы 2 удается точно вычислить. В условиях теоремы 3 его удается описать и доказать, что он отличен от нуля при всех значениях $q$, кроме конечного числа (равного $14 k+20)$.

Мы предполагаем, что гипотезу 1 можно доказать на том же пути, но рассуждения, доказывающие, что коэффициенты при резонансных мономах отличны от нуля, будут существенно более громоздки.

1.2. Физическая мотивировка. Приведем физическую интерпретацию уравнения класса Д.

В 1962 г. Б. Джозефсон теоретически обосновал возможность появления сверхпроводящей компоненты для тока, протекающего через слабый электрический контакт двух сверхпроводящих электродов (такой контакт называется джозефсоновским переходом). Через год существование этого эффекта (названного позже эффектом Джозефсона) было подтверждено экспериментально.

Предположим, что через джозефсоновский переход протекает заданный ток $i(t)=\bar{i}+\tilde{i}(t)$ (например, ток порождается переменным электромагнитным полем - внешним сигналом), который разлагается в сумму постоянного слагаемого $\bar{i}$ и периодического слагаемого $\tilde{i}$ с нулевым средним. Напряжение на электродах джозефсоновского перехода задается производной по времени от функции $\varphi$. В то время как функция $\dot{\varphi}$ описывает макрофизическую величину (напряжение на полюсах ), сама функция $\varphi$ имеет квантово-механическую природу. Это - разность фаз волновых функций, описывающих коллективные свойства «жидкости» куперовских пар электронов в сверхпроводящих электродах.

Для описания поведения джозефсоновского перехода успешно используется так называемая резистивная модель с малой емкостью (большим затуханием), которая задается следующим уравнением в безразмерных переменных ([7], [9]):

$$
\dot{\varphi}+F(\varphi)=i(t)
$$

где $F$ - нечетная $2 \pi$-периодическая функция. В физической литературе функция $F$ называется токо-фазовой зависимостью. Для большинства конкретных реализаций джозефсоновского перехода функция $F$ имеет вид

$$
F(\varphi)=\sin \varphi+H(\varphi)
$$

причем слагаемое $H$ либо тождественно равно нулю, либо мало, и им часто пренебрегают (получающаяся при этом модель хорошо соотносится с результатами эксперимента [11]). Уравнение (5) при малом $H=\delta g$ и малом $\tilde{i}=\varepsilon f$ имеет вид (1) (мы заменяем $\varphi$ на $x$ и $\bar{i}$ на $a$ ). Однако существуют и другие конструкции джозефсоновского перехода, для которых функция $F$ далека от синуса. К ним относится, например, так называемый SNS-сэндвич, с прослойкой из чистого металла при очень низких температурах [9, с. 117].

Зависимость среднего (по времени) значения напряжения $\dot{\varphi}$ от среднего значения тока в уравнении (5) называется вольт-амперной характеристикой джозефсоновского перехода. Для перехода, описываемого уравнением (5), эта характеристика является числом вращения отображсения Пуанкаре уравнения (5) за время $2 \pi$, рассматриваемого как функция от параметра $a$ при фиксированных $\varepsilon$ и $\delta$; ниже эта функция обозначается через $\rho$. Действительно, среднее 
значение напряжения за время $T$ - это значение фазы $\varphi(T)$, деленное на время $T$. По определению это число вращения уравнения (5).

В типичных семействах диффеоморфизмов окружности рациональные числа вращения принимаются на целых отрезках значений параметров. Соответствующие ступеньки на графике функции $\rho$ в физической литературе называются ступеньками Шапиро.

Как сказано выше, для уравнения (1) при $\delta=0$ ступеньки Шапиро появляются только при целых значениях числа вращения. В работе [4] это явление названо квантованием числа вращения. Наши основные результаты, теоремы 2,3 и 6, показывают, что при возмущении синусоидальной токо-фазовой зависимости ступеньки Шапиро имеют точки накопления и могут быть даже всюду плотны на вольт-амперной характеристике.

Таким образом, джозефсоновские контакты с токо-фазовой зависимостью, описывающейся уравнениями класса Д, с одной стороны, и возмущениями этого уравнения, с другой, обладают совершенно различными вольт-амперными характеристиками. Тем самым, физически значимый эффект отсутствия ступенек Шапиро, отвечающих дробным значениям числа вращения, описывается семейством уравнений, имеющим коразмерность бесконечность в пространстве всех уравнений (5). Мы хотим подчеркнуть парадоксальность этого явления в свете философии «общности положения», восходящей к Арнольду и Тому.

1.3. План статьи. Элементарное доказательство теоремы 1 приведено в 2 . Там же предложен простой алгоритм исследования границы зон захвата фазы в семействе уравнений класса Д. Теорема 2 доказана в разд. 5.1, который предваряется выводом выпрямляющей замены (§3) и сведениями из теории Галкина (§4). Теорема 3 доказана в разд. 5.2. В последнем разделе из нее выведена теорема 6, которая устанавливает существование плотного множества языков Арнольда для наиболее общего возмущения семейства (1).

Мы благодарим В. М. Бухштабера, который обратил наше внимание на уравнение класса Д и, в частности, на работы [2]-[4], [8], [11].

\section{§2. Уравнение класса Д как динамическая система на группе $S L_{2}$ и границы зон захвата фазы}

Начнем с доказательства теоремы 1. Как было объяснено во введении, она вытекает из следующего факта (полученного также в работах [10], [12]):

Теорема 4. Отображение Пуанкаре уравнения (2) аналитически эквивалентно дробно-линейному преобразованию.

Доказательство. Замена $y=\operatorname{tg}(x / 2)$ переводит окружность $S^{1}=\mathbb{R} / 2 \pi \mathbb{Z}$ в проективную прямую. Уравнение (2) принимает вид

$$
\dot{y}=y+\frac{1}{2}(a+\varepsilon f(t))\left(y^{2}+1\right) \text {. }
$$

Это уравнение Риккати с периодическими коэффициентами. Хорошо известно, что оно является проективизацией линейной системы. Его преобразование монодромии за период дробно-линейно. Это доказывает теорему 4 и вместе с ней теорему 1.

Опишем теперь границу зоны захвата фазы для уравнения (2). Для этого рассмотрим линейную систему, проективизацией которой является уравнение (7):

$$
\dot{X}=A(t) X, \quad X \in S L(2, \mathbb{R}), A(t) \in \operatorname{sl}(2, \mathbb{R}),
$$


где

$$
2 A(t)=\left(\begin{array}{cc}
1 & a+\varepsilon f(t) \\
-(a+\varepsilon f(t)) & -1
\end{array}\right) .
$$

Пусть $X$ - фундаментальная матрица решений этой системы с начальным условием $X(0)=E$,

$$
X(t)=\left(\begin{array}{ll}
a_{1}(t) & a_{2}(t) \\
a_{3}(t) & a_{4}(t)
\end{array}\right) .
$$

Теорема 5. Граница зоны захвата фазы в уравнении (2) задается уравнением

$$
\operatorname{tr} X(2 \pi)= \pm 2
$$

Доказательство. Перейдем от уравнения (2) к уравнению (7). Оно является проективизацией системы (8), (9). Поэтому отображение Пуанкаре уравнения (7) за период имеет вид

$$
P(y)=\frac{a_{1}(2 \pi) y+a_{2}(2 \pi)}{a_{3}(2 \pi) y+a_{4}(2 \pi)} .
$$

В системе $(8),(9) \operatorname{tr} A(t) \equiv 0$. Поэтому $\operatorname{det} X(t) \equiv 1$ по формуле ЛиувилляОстроградского. Отображение $P$ является гиперболическим, параболическим или эллиптическим, если и только если матрица $A(2 \pi)$ имеет соответственно вещественные и различные, кратные или комплексные (невещественные) собственные значения. Это доказывает теорему 5.

Отметим, что для типичной функции $f$ уравнение (8) неинтегрируемо. Поэтому на практике границу зоны захвата фазы в пространстве параметров можно находить следующим образом. Функцию $f$, зависящую от параметров и входящую в уравнение (2), подставляем в уравнение (8), (9) и находим след $\operatorname{tr} X(2 \pi)$ как функцию параметров. Уравнение интересующей нас границы имеет вид (11).

\section{§3. Выпрямляющая замена для уравнения класса Д и его возмущений}

Всюду в дальнейшем мы рассматриваем уравнение (1) при $a>1$. Случай $a<-1$ сводится к этому обращением времени.

3.1. Интегрируемый случай. Рассмотрим уравнение (1) при $\varepsilon=\delta=0$ (т. е. уравнение (4)):

$$
\dot{x}=\sin x+a
$$

При $a>1$ оно не имеет особых точек, и поэтому соответствующее векторное поле на окружности выпрямляется: существует такая замена переменной $\alpha=$ $\alpha(x)$, что $\dot{\alpha}=$ const.

Пусть

$$
\lambda:=\sqrt{a^{2}-1}, \quad a=\sqrt{\lambda^{2}+1} .
$$


Предложение 1. Выпрямляющая замена для уравнения (4) может быть представлена в виде

$$
\begin{aligned}
\alpha & =\frac{1}{i} \ln \left(b \frac{i e^{i x}-b^{-1}}{i e^{i x}-b}\right), \\
x & =\frac{1}{i} \ln \left(-i b \frac{u-b^{-1}}{u-b}\right),
\end{aligned}
$$

где

$$
u:=e^{i \alpha}, \quad b:=a+\lambda=1 /(a-\lambda)>1 .
$$

При этой замене переменной уравнение (4) принимает вид $\dot{\alpha}=\lambda$.

Следствие 1. При $\varepsilon=\delta=0$ число вращения отображения Пуанкаре уравнения (1) равно $\lambda=\sqrt{a^{2}-1}$.

Доказательство предложения 1. Заметим, что любая выпрямляющая замена имеет вид

$$
\alpha=2 \pi \frac{I(x)}{I(2 \pi)}+C, \quad \text { где } I(x)=\int_{0}^{x} \frac{d y}{\sin y+a} .
$$

Поскольку

$$
\int \frac{d y}{\sin y+a}=\frac{1}{i \lambda} \ln \left(b \frac{i e^{i x}-b^{-1}}{i e^{i x}-b}\right)+C
$$

(что можно непосредственно проверить дифференцированием), а приращение логарифма при обходе аргумента вокруг 0 равно $2 \pi i$, получаем, что $I(2 \pi)=$ $2 \pi / \lambda$, а также что замена переменной (14) действительно выпрямляющая. Выразив в ней $x$ через $\alpha$, получаем формулу (15).

3.2. Преобразования возмущений. Запишем уравнение (3) в выпрямляющих координатах. Начнем со следующего предложения:

Предложение 2. При замене переменной (15) уравнение $\dot{x}=h(x, t)$ принимает вид

$$
\dot{\alpha}=H(\alpha) h(x(\alpha), t), \quad H(\alpha)=-\frac{(u-b)\left(u-b^{-1}\right)}{2 \lambda u} .
$$

Доказательство. Для доказательства достаточно продифференцировать формулу (15):

$$
\dot{x}=x_{u}^{\prime} u_{\alpha}^{\prime} \dot{\alpha}=-\frac{b-b^{-1}}{i(u-b)\left(u-b^{-1}\right)} i u \dot{\alpha},
$$

откуда и следует требуемое.

Следствие 2. При замене переменной (15) семейство (3), в котором $|a|>1$, переходит в семейство

$$
\dot{\alpha}=\lambda+\varepsilon F_{l}(\alpha, t)+\delta G_{k}(\alpha),
$$

где

$$
\begin{aligned}
F_{l}(\alpha, t) & :=\frac{1}{2 i}\left(v^{l}-v^{-l}\right) H(\alpha), & v & :=e^{i t}, \\
G_{k}(\alpha) & :=\frac{1}{2 i}\left(U^{k}-U^{-k}\right) H(\alpha), & U & =e^{i x(\alpha)} .
\end{aligned}
$$


Для дальнейших вычислений нам понадобятся коэффициенты Фурье функций $F_{l}$ и $G_{k}$. Разложение в ряд Фурье любой функции $\Phi$ на торе мы будем записывать в виде

$$
\Phi(\alpha, t)=\sum[\Phi]_{m, n} u^{m} v^{n} .
$$

Носитель этого ряда - это множество

$$
\sigma(\Phi)=\left\{(m, n) \in \mathbb{Z}^{2} \mid[\Phi]_{m, n} \neq 0\right\} .
$$

Предложение 3. (i) Функиия $F_{l}$ является тригонометрическим полиномом с носителем $\sigma F_{l}=\{0, \pm 1\} \times\{ \pm l\}$,

$$
F_{l}(\alpha, t)=f_{0} \cdot\left(v^{l}-v^{-l}\right)\left(u-2 a+u^{-1}\right), \quad f_{0}=\frac{i}{4 \lambda} \neq 0 .
$$

(ii) Носитель бункиии $G_{k}$ принадлежст множеству $\mathbb{Z} \times\{0\}$ при любом $k$. При $k=2,|q| \geqslant 2$

$$
\left[G_{k}\right]_{q, 0}=g_{0} b^{-|q|}, \quad g_{0}=2 i \lambda^{2} \neq 0 .
$$

Знаменатели рациональных функций в формуле (19) равны $(u-b)^{k-1}$ и $\left(u-b^{-1}\right)^{k-1}$. Действительно, как немедленно следует из предложения 2 , при замене (15) уравнение $\dot{x}=e^{i k x}$ принимает вид

$$
\dot{\alpha}=-\frac{(u-b)\left(u-b^{-1}\right)}{2 \lambda u} \exp \left(k \ln \left(-i b \frac{u-b^{-1}}{u-b}\right)\right)=-\frac{(-i b)^{k}}{2 \lambda u} \frac{\left(u-b^{-1}\right)^{k+1}}{(u-b)^{k-1}} .
$$

Поэтому при $k=2$ формула для $G_{k}$ особенно проста. Коэффициенты Фурье функции $G_{k}$ при $k>2$ вычислены в разд. 5.3.

Доказательство предложения 3. Раскроем скобки в формуле (16) и заметим, что $H(\alpha)=-\frac{1}{2 \lambda}\left(u-2 a+u^{-1}\right)$. Подставив это выражение в формулу (18), получаем утверждение (i).

Для доказательства утверждения (ii) воспользуемся формулой (23). При $k=2$ уравнение $\dot{x}=\sin k x$ принимает вид

$$
\dot{\alpha}=\frac{1}{4 i \lambda u}\left(\frac{b^{2}\left(u-b^{-1}\right)^{3}}{u-b}-\frac{b^{-2}(u-b)^{3}}{u-b^{-1}}\right) .
$$

Учитывая, что $|u|=1, b>1$, имеем

$$
\begin{gathered}
\frac{1}{u-b}=-\frac{1}{b\left(1-\frac{u}{b}\right)}=-b^{-1} \sum_{m=0}^{+\infty} u^{m} b^{-m} \\
\frac{1}{u-b^{-1}}=-\frac{1}{u\left(1-\frac{1}{u^{-1} b^{-1}}\right)}=-u^{-1} \sum_{m=0}^{+\infty} u^{-m} b^{-m}=-b \sum_{m=-\infty}^{-1} u^{m} b^{m} .
\end{gathered}
$$

Осталось подставить полученные выражения в формулу (24) и перегруппировать слагаемые, суммируя коэффициенты при $u^{m}$ :

$$
\dot{\alpha}=\frac{1}{4 i \lambda}\left[-\left(u-b^{-1}\right)^{3} \sum_{m=-1}^{+\infty} u^{m} b^{-m}+(u-b)^{3} \sum_{m=-\infty}^{-2} u^{m} b^{m}\right] .
$$

Ясно, что при $|m| \geqslant 2$ в формулу для коэффициента при $u^{m}$ входят слагаемые лишь от одной из сумм, поэтому коэффициенты $\left[G_{2}\right]_{m, 0}$ при положительных 
и отрицательных $m$ образуют две геометрические прогрессии со знаменателем $b^{-1}<1$ каждая. Собирая коэффициенты при $u^{m}$, в обоих случаях получаем $g_{0}=\frac{1}{4 i \lambda}\left(-\left(b-b^{-1}\right)^{3}\right)=2 i \lambda^{2}$, что и доказывает $(22)$.

Замечание 1. Формула (25), кроме того, дает

$$
\left[G_{2}\right]_{1,0}=g_{0} b^{-1}+\frac{b^{2}+b^{-2}}{4 i \lambda}=g_{0} b^{-1}\left(1+\frac{b\left(1-2 a^{2}\right)}{4 \lambda^{3}}\right) .
$$

\section{§4. Захват фазы}

В этом параграфе излагаются результаты классической теории возмущений в форме, необходимой для дальнейшего.

4.1. Критерий захвата. Рассмотрим аналитическое уравнение на торе

$$
\dot{\alpha}=\lambda+\varepsilon F+\delta G .
$$

Обозначим через $\rho(\lambda, \varepsilon, \delta)$ его число вращения, а через $P(\cdot, \lambda, \varepsilon, \delta)-$ соответствующее отображение Пуанкаре.

Предположим, что при $\lambda=p / q$ и малых $\varepsilon, \delta$ в семействе $(27)$ не происходит захвата фазы. Тогда существует аналитическая функция $\lambda(\varepsilon, \delta)$, такая, что $\lambda(0,0)=p / q, \rho(\lambda(\varepsilon, \delta)) \equiv p / q, P^{q}(\alpha, \lambda(\varepsilon, \delta), \varepsilon, \delta) \equiv \mathrm{id}$. В этом случае уравнение

$$
\dot{\alpha}=\lambda(\varepsilon, \delta)+\varepsilon F+\delta G
$$

послойно (с сохранением времени) при всех малых $\varepsilon, \delta$ сопряжено уравнению

$$
\dot{\beta}=p / q .
$$

Таким образом, если сопряжения нет, то захват фазы есть.

4.2. Методы теории возмущений. Итак, наличие захвата фазы следует из неразрешимости следующей задачи: найти функцию

$$
\lambda(\varepsilon, \delta)=\frac{p}{q}+\sum_{m+n=1}^{\infty} \varepsilon^{m} \delta^{n} \lambda_{m n}
$$

и сопряжение $\alpha(\beta, t)=\beta+\sum_{m+n=1}^{\infty} \varepsilon^{m} \delta^{n} h_{m n}(\beta, t)$, которое переводит семейство (28) в уравнение (29). Соответствующее функциональное уравнение имеет вид

$$
\begin{aligned}
\frac{p}{q}+\sum_{m+n=1}^{\infty} \varepsilon^{m} \delta^{n} L_{\omega} h_{m n}(\beta, t) & =\frac{p}{q}+\sum_{m+n=1}^{\infty} \varepsilon^{m} \delta^{n} \lambda_{m n} \\
& +\varepsilon F\left(\beta+\sum_{m+n=1}^{\infty} \varepsilon^{m} \delta^{n} h_{m n}, t\right)+\delta G\left(\beta+\sum_{m+n=1}^{\infty} \varepsilon^{m} \delta^{n} h_{m n}, t\right) .
\end{aligned}
$$

Приравнивая коэффициенты при одинаковых степенях $\varepsilon, \delta$ в уравнении (30), мы получим бесконечную серию уравнений вида

$$
L_{\omega} h_{m n}=F_{m n} .
$$

Для разрешимости каждого такого уравнения необходимо, чтобы среднее значение правой части было равно нулю. Это требование определяет коэффициент $\lambda_{m n}$. 
Для каждой функции $\Phi$ на окружности обозначим через $\bar{\Phi}$ ее среднее значение, а через $\widetilde{\Phi}$ разность $\Phi-\bar{\Phi}$. Приравнивая в уравнении $(30)$ коэффициенты при одинаковых степенях $\varepsilon, \delta$, получаем

$$
\begin{aligned}
& L_{\omega} h_{10}=\widetilde{F}, \\
& L_{\omega} h_{01}=\widetilde{G}, \\
& L_{\omega} h_{20}=\widetilde{F_{\beta} h_{10}}, \\
& L_{\omega} h_{11}=\widetilde{F_{\beta} h_{01}}+\widetilde{G_{\beta} h_{10}}, \\
& L_{\omega} h_{02}=\widetilde{G_{\beta} h_{01}}, \\
& \ldots .
\end{aligned}
$$

Напомним, что если эта система при $\omega=(p / q, 1)$ неразрешима, то захват фазы в семействе $(27)$ при $\lambda=p / q$ происходит, и на графике числа вращения как функции от $\lambda$ при фиксированных ненулевых $\varepsilon$ и $\delta$ возникает ступенька.

4.3. Резонансные мономы. Напомним хорошо известные результаты о разрешимости системы $(31)$. Пусть, как и выше, $[h]_{m, n}$ обозначает коэффициент Фурье при мономе $u^{m} v^{n}$ в разложении функции $h=h(\alpha, t)$ в ряд Фурье, $(m, n) \neq(0,0)$. Тогда:

1. $\left[h_{\beta}\right]_{m, n}=i m[h]_{m, n}$.

2. Если $L_{\omega} h=f$, то $[f]_{m, n}=i(m \lambda+n)[h]_{m, n}$.

3. Если $L_{\omega} h=f$ и $m \lambda+n \neq 0$, то $[h]_{m, n}=\frac{[f]_{m, n}}{i(m \lambda+n)}$. Если же $m \lambda+n=0$, а $[f]_{m n} \neq 0$, то уравнение $L_{\omega} h=f$ неразрешимо. Напротив, если $[f]_{m n}=0$, то коэффициент $[h]_{m n}$ можно выбрать произвольно.

Таким образом, если коэффициент Фурье хотя бы при одном резонансном (т.е. таком, что $m p+n q=0$ ) мономе в правой части одного из уравнений системы (31) не равен нулю, то соответствующее уравнение неразрешимо.

Следовательно, послойная эквивалентность систем (27) и (29) при рациональном числе вращения равносильна обращению в нуль коэффищиентов при всех резонансных мономах в системе (31). Это представляется крайне маловероятным, однако происходит, когда отображение Пуанкаре эквивалентно дробно-линейному. Мы получили следующий

Критерий захвата фазы для системы (27). B системе (27) с рациональным значением $\lambda$ происходит захват фазы, если и только если в правой части хотя бы одного из уравнений системы (31) возникает резонансный моном с ненулевым коэфбициентом.

4.4. Приложение к возмущенному уравнению класса Д. Пусть $\sigma F-$ носитель ряда Фурье для $F$. Заметим, что носитель ряда $h$ для решения уравнения $L_{\omega} h=w$ совпадает с носителем ряда $w$ (если, конечно, уравнение разрешимо). Кроме того, при дифференцировании носитель ряда не расширяется, а носитель произведения рядов принадлежит сумме их носителей как множеств. Таким образом, для решений системы (31) справедливы следующие равенства и включения:

$$
\sigma h_{10}=\sigma F, \quad \sigma h_{01}=\sigma G, \quad \sigma h_{20} \subset \sigma F+\sigma F, \quad \sigma h_{02} \subset \sigma G+\sigma G,
$$

где $A+B=\{a+b: a \in A, b \in B\}$. Поэтому правые части всех выписанных уравнений системы (31), кроме четвертого, не содержат резонансных мономов. 
Напротив,

$$
\sigma h_{11} \subset \sigma F+\sigma G,
$$

и, как показывает рисунок, есть три пути к резонансному моному с показателем $(q,-l)$.

Каждый из этих путей состоит из двух векторов, принадлежащих $\sigma F$ и $\sigma G$. Теория Галкина [5] позволяет заключить, что наличие ненулевых резонансных мономов в правой части уравнения на $h_{11}$ системы $(31)$ влечет за собой существование языка Арнольда, ширина которого имеет порядок $\varepsilon \delta$.

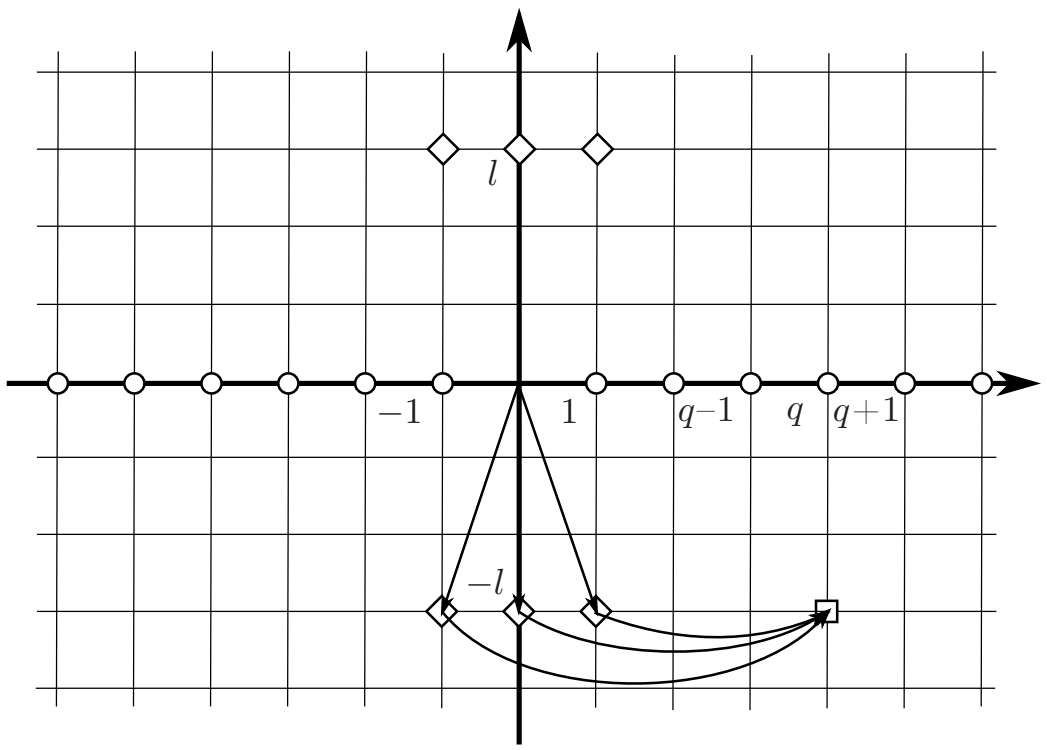

Рис. 1. Три представления для вектора $(q,-l)$ в виде суммы векторов из $\sigma F$ и $\sigma G$

При этом, очевидно, отмеченный выше произвол в выборе коэффициентов при резонансных мономах при решении системы (31) никак не влияет на разрешимость четвертого уравнения из (31). Поэтому

$$
\left[h_{11}\right]_{q,-l}=D_{-1}+D_{0}+D_{1},
$$

где

$$
D_{j}=\left[h_{01}\right]_{q+j, 0}\left[F_{\beta}\right]_{-j,-l}+\left[G_{\beta}\right]_{q+j, 0}\left[h_{10}\right]_{-j,-l} .
$$

Согласно формулам из предыдущего раздела,

$$
\left[h_{01}\right]_{a, b}\left[F_{\beta}\right]_{c, d}+\left[G_{\beta}\right]_{a, b}\left[h_{10}\right]_{c, d}=[G]_{a, b}[F]_{c, d}\left(\frac{c}{a \lambda+b}+\frac{a}{c \lambda+d}\right) .
$$

Отсюда следует, что

$$
D_{j}=[G]_{q+j, 0}[F]_{-j,-l}\left(\frac{-j}{(q+j) \lambda}+\frac{q+j}{-j \lambda-l}\right)=-[G]_{q+j, 0}[F]_{-j,-l} \frac{q(q+2 j)}{l(q+j)} .
$$

По формуле (21)

$$
F_{-1,-l}=F_{1,-l}=-f_{0}, \quad F_{0,-l}=2 a f_{0} .
$$


Заменим для краткости $[G]_{m, 0}$ на $[G]_{m}$. Получим

$$
\left[h_{11}\right]_{q,-l}=-f_{0} \frac{q}{l}\left([G]_{q-1} \frac{q-2}{q-1}-[G]_{q} 2 a+[G]_{q+1} \frac{q+2}{q+1}\right) .
$$

Это заканчивает вычисление коэффициента при интересующем нас резонансном мономе. В следующем параграфе мы доказываем, что этот коэффициент для рассматриваемых нами возмущений (как правило) отличен от нуля.

\section{§5. Накопление языков Арнольда}

Здесь мы завершаем доказательство основных результатов статьи.

\section{1. Языки Арнольда: случай $k=2$.}

Доказательство теоремы 2. Из предложения 3 немедленно вытекает, что при выпрямляющей замене (15) семейство (3) при $k=2$ принимает вид

$$
\dot{\alpha}=\lambda+\varepsilon F(\alpha, t)+\delta G(\alpha),
$$

где функции $F(\alpha, t)=F_{l}(\alpha, t)$ и $G(\alpha)=G_{2}(\alpha)$ задаются равенствами $(21),(22)$.

Докажем, что для всех натуральных $q>1$ при $\lambda=l / q$ коэффициент при резонансном мономе с показателем $(q,-l)$ в правой части четвертого уравнения системы (31) ненулевой. (В силу симметрии при отрицательном $q$ коэффициент при резонансном мономе с тем же показателем также будет ненулевым.) Тогда теорема будет следовать из критерия захвата, полученного в разд. 4.3.

По формулам (22), (35) при $k=2$ и $|q|>2$ получаем

$$
\left[h_{11}\right]_{q,-l}=-f_{0} g_{0} \frac{q}{l} b^{-|q|}\left(b \frac{q-2}{q-1}-2 a+\frac{q+2}{b(q+1)}\right) .
$$

Множитель перед скобкой не равен нулю, выражение в скобке - тоже. Действительно, это выражение, умноженное на $b\left(q^{2}-1\right)$, равно в силу (13) и равенства $b^{2}-2 a b+1=0$ следующему:

$$
b^{2}(q-2)(q+1)-2 a b\left(q^{2}-1\right)+(q+2)(q-1)=-2 b(a+l) \neq 0
$$

при $|q|>2$.

Согласно формуле (26), при $q=2$ для вычисления коэффициента при резонансном мономе в выражении (37) нужно умножить первое слагаемое на $1+b\left(1-2 a^{2}\right) /\left(4 \lambda^{3}\right)$, поэтому коэффициент $\left[h_{11}\right]_{ \pm 2,-l}$ также оказывается ненулевым.

\section{2. Языки Арнольда: случай $k>2$.}

Доказательство теоремы 3. Для доказательства теоремы 3 достаточно установить, что коэффициент Фурье при резонансном мономе $v^{-l} u^{q}$ в разложении функции $h_{11}$ отличен от нуля. Ниже мы докажем, что этот коэффициент, обозначенный выше через $\left[h_{11}\right]_{q,-l}$ и рассматриваемый как функция от $q$ при фиксированном $l$, имеет не больше чем $14 k+20$ нулей.

Функция $h_{11}$ зависит от $k$ и $l$ как от параметров; тем же свойством обладают ее коэффициенты Фурье. Следующее обозначение подчеркивает зависимость от $k$ при фиксированном $l$ интересующего нас коэффициента Фурье. Положим $\varphi_{k}(q)=\left[h_{11}\right]_{q,-l}$. Коэффициент $\varphi_{k}(q)$ выражен в (35) через коэффициенты 
Фурье возмущений $F$ и $G$. Возмущение $F$ зависит только от $l$, см. (18); возмущение $G$ зависит от $k$, и мы вернемся к обозначению $G_{k}$ вместо $G$, см. (19). Найдем коэффициенты Фурье функции $G_{k}$ при $k>2$.

По формуле (23)

$$
G_{k}(\alpha)=\frac{1}{4 i \lambda u}\left[\frac{(-i b)^{k}\left(u-b^{-1}\right)^{k+1}}{(u-b)^{k-1}}-\frac{(i b)^{k}(u-b)^{k+1}}{\left(u-b^{-1}\right)^{k-1}}\right]=: \widehat{G}_{k}(u),
$$

где $u=e^{i \alpha}$. Коэффициенты Фурье функции $G_{k}$ - это коэффициенты разложения функции $\widehat{G}_{k}$ в ряд Лорана в кольце $b^{-1}<|u|<b$. Они зависят от $k, l$ и $q$ как от параметров, но мы не указываем эту зависимость в обозначениях. Элементарные вычисления дают

$$
\widehat{G}_{k}(u)=-g_{0} \sum_{m=0}^{\infty} b^{-m} D_{m}\left(u^{m}+(-1)^{k} u^{-m}\right),
$$

где при $m \geqslant k$ функции $D_{m}$ задаются формулой

$$
D_{m}=\sum_{n=0}^{k+1} C_{k+1}^{n} C_{m-n+k-1}^{m-n+1}\left(-b^{2}\right)^{n}
$$

и $g_{0}:=\left(4 \lambda(i b)^{k+1}\right)^{-1} \neq 0$.

Таким образом, коэффициенты Фурье функции $G_{k}$ имеют вид

$$
\left[G_{k}\right]_{m, 0}=-g_{0} b^{-|m|} D_{|m|},
$$

где при $|m| \geqslant k$ коэффициенты $D_{|m|}$ определены в формуле (39), а

$$
b=b^{(q)}=\lambda+\sqrt{\lambda^{2}+1}=\frac{\sqrt{l^{2}+q^{2}}+l}{q}=: \frac{s(q)}{q} .
$$

Подставляя полученное значение $\left[G_{k}\right]_{m}$ при $m \in\{q-1, q, q+1\}, q \geqslant k+1$, в формулу (35), получаем

$$
\varphi_{k}(q)=-f_{0} g_{0} \frac{q}{l} b^{-|q|} r_{k}(q),
$$

где $b f_{0} g_{0} \neq 0$ и при $|q| \geqslant k+1$ функция $r_{k}$ имеет вид

$$
r_{k}(q):=D_{q-1} b \frac{q-2}{q-1}-2 a D_{q}+D_{q+1} \frac{q+2}{b(q+1)} .
$$

Утверждение теоремы вытекает теперь из следующего предложения:

Предложение 4. Уравнение $\varphi_{k}(q)=0$ имеет не более $7 k+10$ натуральных решений.

Доказательство. При $q \geqslant k+1$ функции $\varphi_{k}$ и $r_{k}$ имеют вид (42) и (43). Продолжим функции $\varphi_{k}$ и $r_{k}$, заданные этими формулами, на всю вещественную ось $q$; продолженные функции обозначим через $\tilde{\varphi}_{k}, \tilde{r}_{k}$. Докажем, что $\tilde{r}_{k}-$ алгебраическая функция, и оценим сверху число ее нулей. Преобразуем выражения (39) для $D_{m}$, пользуясь (41):

$$
\widehat{D}_{m}(q):=q^{2(k+1)} D_{m}(q)=\sum_{0}^{k+1} C_{k+1}^{n} C_{m-n+k-1}^{m-n+1}\left(-s^{2}\right)^{n} q^{2(k+1-n)} .
$$


Подставляя в (43) выражения для $\widehat{D}_{m}$ при $m=q-1, q, q+1$ и $b(q)$ из $(41)$, получаем

$$
q^{2(k+1)}\left(q^{2}-1\right) s(q) q \tilde{r}_{k}(q)=T_{k}(q),
$$

где $T_{k}(q)=\widehat{T}_{k}(q, s(q))$,

$$
\widehat{T}_{k}(q, s)=\widehat{D}_{q-1} s^{2}(q-2)(q+1)-2 \widehat{D}_{q}(s-l) s\left(q^{2}-1\right)+\widehat{D}_{q+1} q^{2}(q+2)(q-1) .
$$

Функция $\widehat{D}_{m}$ при любом $m$ является полиномом от $q$ и $s(q)$ степени $3 k$, поскольку $C_{q-n+k-1}^{q-n+1}-$ полином от $q$ степени $k-2$. Следовательно, $\widehat{T}_{k}-$ полином от $q$ и $s$ степени $3 k+4$. Так как $s^{2}=2 l^{2}+q^{2}+2 l \sqrt{l^{2}+q^{2}}$, функцию $T_{k}$ можно представить в виде

$$
T_{k}(q)=P(q)+\sqrt{l^{2}+q^{2}} R(q),
$$

где $P$ и $R$ - полиномы степени не выше чем $3 k+4$. Уравнение $T_{k}(q)=0$ эквивалентно системе

$$
P+t R=0, \quad t^{2}=q^{2}+l^{2} .
$$

По теореме Безу эта система имеет не более чем $2(3 k+5)$ решений при условии, что $T \not \equiv 0$. Чтобы проверить последнее, убедимся, что $T(0) \neq 0$. Отметим, что $s(0)=2 l$. По формуле $(44)$

$$
T_{k}(0)=\widehat{D}_{-1}(0)\left(-8 l^{2}\right)+\widehat{D}_{0}(0) \cdot 4 l^{2} .
$$

Далее, все слагаемые при $n \neq k+1$ в выражении для $\widehat{D}_{m}(0)$ равны нулю. Поэтому

$$
\begin{gathered}
\widehat{D}_{-1}(0)=\left(-4 l^{2}\right)^{k+1} C_{-3}^{k-1}, \quad C_{-3}^{k-1}=\prod_{-3}^{-(k+1)} j, \\
\widehat{D}_{0}(0)=\left(-4 l^{2}\right)^{k+1} C_{-2}^{k}, \quad C_{-2}^{k}=\prod_{-2}^{-k} j, \\
T_{k}(0)=-\left(-4 l^{2}\right)^{k+2}\left(\prod_{-3}^{-k} j\right)(2 k+5) \neq 0 .
\end{gathered}
$$

Таким образом, на множестве $q \geqslant k+1$ функция $\tilde{\varphi}_{k}$ имеет не более чем $6 k+10$ нулей. Следовательно, на множестве всех натуральных $q$ функция $\tilde{\varphi}_{k}$ (а значит, $\left.\varphi_{k}\right)$ имеет не более чем $7 k+10$ нулей.

Из предложения 4 следует, что коэффициент при резонансном мономе $\left[h_{11}\right]_{q,-l}$ отличен от нуля для всех положительных $q$, кроме, быть может, $7 k+10$ значений. В силу симметричности формулы (38) для отрицательных $q$ выполняется та же оценка. Это завершает доказательство теоремы 3.

5.3. Захват фазы в уравнениях класса Д, возмущенных периодической функцией от времени общего вида. Теоремы 2 и 3 остаются верными и в случае $f(t)=A \sin l t+B \cos l t$ (если $A$ и $B$ не равны нулю одновременно). Действительно, изменения в коэффициентах при резонансных мономах сводятся к домножению на $A+i B$, если степень $v$ положительна, и на $A-i B$, если она отрицательна. Поэтому коэффициенты при резонансных мономах остаются ненулевыми.

Пусть теперь

$$
f(t)=\sum_{1}^{\infty} a_{l} \sin l t+b_{l} \cos l t
$$


Для такой функции $f$ семейство (1) при выпрямляющей замене (44) переходит в семейство (27). Носитель функции $F$ в правой части этого семейства расположен на прямых $q= \pm 1, q=0$. Коэффициент Фурье $\left[h_{11}\right]_{q,-p}$ не зависит от коэффициентов $a_{l}, b_{l}$ при $l \neq p$ и выражается через коэффициенты $a_{p}, b_{p}$ по тем же формулам, что и раньше, т. е. в случае, когда $a_{l}=b_{l}=0$ при $l \neq \pm p$. Отсюда следует

Теорема 6. Пусть в семействе (1) функиия $f$ имеет вид (45), a $g=\sin k x$, $|k|>1$. Тогда для типичного набора параметров $a_{l}, b_{l}$ захват фазы в семействе (1) происходит при всех рациональных числах вращения $p / q$ (кроме, быть может, $14 k+20$ значений q при каждом фиксированном p). Соответствующие языки Арнольда имеют ширину порядка $\varepsilon \delta$.

\section{ЛитературА}

[1] В. И. Арнольд, Дополнительные главы теории обыкновенных дифберенциальных уравнений, Наука, М., 1978.

[2] В. М. Бухштабер, О. В. Карпов, С. И. Тертычный, О свойствах дифберенциального уравнения, описывающего динамику сильношунтированного перехода Дюсозефсона, УМН, 59:2 (2004), 187-188.

[3] В. М. Бухштабер, О. В. Карпов, С. И. Тертычный, Математические модели динамики силъношунтированного перехода Джозефсона, УМН, 63:3 (2008), 155-156.

[4] В. М. Бухштабер, О. В. Карпов, С. И. Тертычный, Эфбект квантования числа вращения, ТМФ, 162:2 (2010), 254-265.

[5] О. Г. Галкин, Фазовый захват для отображений тора типа Матъе, Функц. анализ и его прил., 27:1 (1993), 1-11.

[6] Ю. С. Ильяшенко, Лекиии по динамическим системам, Летняя школа-2009, не опубликовано.

[7] К. К. Лихарев, Б. Т. Ульрих, Системы с джозефсоновскими контактами, Изд-во МГУ, М., 1978.

[8] С. И. Тертычный, Об асимптотических свойствах решений уравнения $\dot{\phi}+$ $\sin \phi=f$ при периодическом $f$, УМН, 55:1 (2000), 195-196.

[9] В. В. Шмидт, Введение в физику сверхпроводников, МЦНМО, М., 2000.

[10] R. L. Foote, Geometry of the Prytz planimeter, Rep. Math. Phys., 42:1-2 (1998), 249-271.

[11] O. V. Karpov, V. M. Buchstaber, S. I. Tertychniy, J. Niemeyer, O. Kieler, Modeling of rf-biased overdamped Josephson junctions, J. Appl. Phys., 104:9 (2008), 093910.

[12] M. Levi, S. Tabachnikov, On bicycle tire tracks geometry, hatchet planimeter, Menzin's conjecture and oscillation of unicycle tracks, Experiment. Math., 18:2 (2009), 173-186.

[13] S. A. Marvel, R. E. Mirollo, S. H. Strogatz, Identical phase oscillators with global sinusoidal coupling evolve by Möbius group action, Chaos, 19, no. 4 (2009).

Корнельский университет, США,

Московский государственный университет,

Поступило в редакцию

Независимый московский университет,

Математический институт им. В. А. Стеклова

e-mail: yulijs@gmail.com

Санкт-Петербургский государственный университет,

лаборатория им. П. Л. Чебышева

e-mail: mitya160@gmail.com

Московский государственный университет путей сообщения

e-mail: mityafi@mccme.ru 